OPEN ACCESS

Edited by:

Sha Wu,

Southern Medical University, China

Reviewed by:

Huang Chunyu,

The University of Hong Kong, Hong Kong, SAR China

Maikel Peppelenbosch,

Erasmus Medical Center, Netherlands

${ }^{*}$ Correspondence:

Yan Zhang

peneyyan@mail.ustc.edu.cn

Tailang Yin

reproductive@whu.edu.cn

${ }^{t}$ These authors have contributed equally to this work

Specialty section:

This article was submitted to Immunological Tolerance and Regulation,

a section of the journal

Frontiers in Immunology

Received: 30 May 2021 Accepted: 05 August 2021 Published: 19 August 2021

Citation:

Wang J, Ding J, Zhang S, Chen X, Yan S, Zhang $Y$ and Yin T (2021) Decreased USP2a Expression Inhibits Trophoblast Invasion and Associates With Recurrent Miscarriage.

Front. Immunol. 12:717370. doi: 10.3389/fimmu.2021.717370

\section{Decreased USP2a Expression Inhibits Trophoblast Invasion and Associates With Recurrent Miscarriage}

\author{
Jiayu Wang ${ }^{1 \dagger}$, Jinli Ding ${ }^{1 \dagger}$, Sainan Zhang ${ }^{1 \dagger}$, Xin Chen ${ }^{1}$, Sisi Yan ${ }^{1}$, Yan Zhang ${ }^{2 *}$ \\ and Tailang Yin ${ }^{1 *}$
}

\footnotetext{
${ }^{1}$ Reproductive Medicine Center, Renmin Hospital of Wuhan University, Wuhan, China, ${ }^{2}$ Department of Clinical Laboratory, Renmin Hospital of Wuhan University, Wuhan, China
}

An appropriate development of the placenta consisting of trophoblast cell migration, invasion, proliferation, and apoptosis, is essential to establishing and maintaining a successful pregnancy. Ubiquitin-specific protease 2a (USP2a) regulates the processes of metastasis in multiple tumor cells. Yet, no known research has focused on exploring the effect of USP2a on trophoblasts and its possible mechanism in the pathogenies of recurrent miscarriage (RM). In this study, we first detected the decreased mRNA levels and the protein levels of USP2a in placental villous tissue samples from the RM patients. In vitro assays verified that overexpression of USP2a promoted human trophoblast proliferation, migration, invasion, whereas knockdown of USP2a inhibited these processes. Mechanistically, USP2a activated PI3K/Akt/GSK3 $\beta$ signaling pathway to promote nuclear translocation of $\beta$-catenin and further activated epithelial-mesenchymal transition (EMT) in the trophoblasts. Moreover, transforming growth factor-beta (TGF- $\beta$ ) up-regulated USP2a expression in trophoblasts. Interestingly, M2 macrophage secreted TGF- $\beta$ induced trophoblast migration and invasion, and an anti-TGF- $\beta$ antibody alleviated this effect. Collectively, this study indicated that USP2a regulated trophoblast invasion and that abnormal USP2a expression might lead to aberrant trophoblast invasion, thus contributing to RM.

Keywords: USP2a, trophoblast invasion, recurrent miscarriage, decidual macrophage, TGF- $\beta$

\section{INTRODUCTION}

RM is defined by the consecutive loss of two or more clinical pregnancies (1) and affects approximately $2-5 \%$ of couples at reproductive age (2). $\mathrm{RM}$ as a severe global health issue, carries a heavy psychological and financial burden for affected couples. As one of the prerequisites for a successful pregnancy, adequate placentation relies on a delicate chorus between fetal trophoblasts and maternal cells, including proper maternal-fetal crosstalk and trophoblasts development (3). Deep invasion of placental trophoblast cells into the maternal decidua and myometrium is essential for placental embedment and fetal development. Epithelialmesenchymal transition (EMT), characterized by loss of adhesive epithelial phenotype and 
acquisition of motile mesenchymal phenotype, has been proven to significantly promote the migration and invasion of extravillous trophoblast cells (EVTs) (4-6). Reduced trophoblast proliferation, excessive trophoblast cell apoptosis, along with insufficient trophoblast invasion have all been tightly linked to the development of RM (7). Thus, exploring trophoblast development is crucial to further understanding RM pathogenesis.

Trophoblast invasion is an intricate process involving interactions with stromal cells, glands, arteries, and immune cells, including decidual natural killer cells, macrophages, dendritic cells, $\mathrm{T}$ cells, and a range of cytokines and chemokines (8). During the establishment and maintenance of pregnancy, the well-orchestrated crosstalk between fetal trophoblasts and maternal immune cells facilitates the formation of a functional placenta (9). Thereby, successful pregnancy requires a robust and highly dynamic immune system. Post-implantation decidua is rich in immune infiltrates (10). Macrophages are the second-largest immune cell group and comprise $20-25 \%$ of all immune cells at the maternalfetal interface $(11,12)$. Accumulating evidence indicates that decidual macrophages are skewed toward an M2-like phenotype involving tissue remodeling, cell proliferation, and generation of immunosuppressive microenvironments in the early pregnancy uterus (12). Previous studies have shown that macrophages can establish "crosstalk" with trophoblasts in the maternal-fetal interface microenvironment via a complex cytokine-based connection. In addition, macrophages can secrete amounts of soluble mediators to regulate the biological behaviors of trophoblasts, including IL-10, TGF- $\beta$, IL-4, and VEGF (13-15). Also, macrophages can respond to various factors produced by trophoblasts to regulate polarization status, thereby exerting different biological functions. Previously, our group has demonstrated that IL-6 secreted by trophoblasts could induce macrophages polarization to M2 phenotype, thereby modulating the process of early pregnancy (16). However, understanding the abnormal interactions between trophoblasts and decidua macrophages at the maternal-fetal interface and its contribution to RM remains limited.

Ubiquitination, as one of the important post-translational modifications, can selectively attach ubiquitin to its substrate to regulate protein degradation, activity, and protein-protein interaction as well (17). The ubiquitin system is highly dynamic and tightly controlled by the family of deubiquitinating enzymes specifically involved in removing ubiquitin molecules on target proteins (18). The deubiquitinating enzyme USP2a inhibits the ubiquitination of FAS, MDM2, cyclin, MYC, Aurora A and regulates their stability and function, thereby participating in the regulation of the metabolism, development, apoptosis, cell proliferation, and differentiation of tumor cells (19-22). Recent studies have shown that USP2a removes the K33-linked ubiquitin chain at Lys502 of TGFBR1 to promote the recruitment and phosphorylation of SMAD2/3, promoting the transcription of EMT-related genes induced by TGF- $\beta$ (23). In addition, USP $2 a$ promotes the nuclear accumulation of $\beta$-catenin and enhances its transcriptional activity by deubiquitinating $\beta$-catenin, and activates the Wnt/ $\beta$-catenin signaling pathway (24). Given the similar regulatory mechanism and biological characteristics of EVTs and tumor cells, we propose whether USP2a participates in the occurrence of RM by regulating the invasive ability of EVTs at the maternal-fetal interface.

Here in the current study, we firstly reported that USP2a facilitated the proliferation, invasion, and migration of trophoblasts by activating the PI3K/Akt/GSK3 $\beta / \beta$-catenin signaling pathway. Furthermore, USP2a expression is regulated positively by TGF- $\beta$ derived from M 2 macrophages. In addition, USP2a expression was down-regulated in chorionic villi of RM patients. Thus, our results suggest that USP2a is a trophoblast proliferation and invasion-associated enzyme that might participate in RM's pathogenesis.

\section{MATERIALS AND METHODS}

\section{Patients and Tissue Samples}

In this study, women with normal pregnancies who chose to undergo an artificial abortion due to their voluntary willingness were selected as the healthy control group (NC). Patients with unexplained recurrent spontaneous abortions two or more times were regarded as the RM group (RM). This study enrolled a total of $24 \mathrm{RM}$ patients treated in Renmin Hospital of Wuhan University and 28 normal pregnant women. All patients denied having a history of chronic diseases such as hypertension and diabetes. RM patients with the following characteristics were excluded: abnormal anatomical structure of the reproductive tract; infection of the reproductive tract; reproductive endocrine disease; prethrombotic state; fetal chromosomal abnormality. The patients in the control group had a history of more than one live birth, and there was no history of adverse pregnancy, such as spontaneous abortion, eclampsia, and preterm birth. These patients received an abortion between 7 and 9 weeks of gestation to terminate the unintended pregnancy. During the operation, samples of placental chorionic and decidual tissues were collected or fixed with $4 \%$ paraformaldehyde and embedded in paraffin in blocks or frozen and stored in liquid nitrogen. The procedure was carried out under the approval of the internal review and ethics committee of the Renmin Hospital of Wuhan University, and the informed consent of all patients was obtained.

\section{Cell Culture and Reagents}

The HTR-8/SVneo cell line and BeWo cell line were obtained from China Center for Type Culture Collection. HTR- 8 and BeWo were respectively cultivated in Dulbecco's modified Eagle's minimal essential medium (DMEM)/F12 (Gibco, USA) and Minimum Essential Medium Eagle medium (MEM) (Gibco, USA) with $10 \%$ fetal bovine serum (FBS) (Gibco, USA) and $1 \%$ antibiotic, at $37^{\circ} \mathrm{C}$ in the presence of $5 \% \mathrm{CO}_{2}$.

For macrophage generation, the human monocyte cell line THP-1 was cultured and grown in RPMI-1640 medium (Gibco, USA) with $10 \%$ FBS. THP-1 cells were treated with $100 \mathrm{ng} / \mathrm{ml}$ Phorbol1 2-myristate 13-acetate (PMA; Sigma, USA) for $24 \mathrm{~h}$. Macrophages were polarized in M1 macrophages by incubation with $20 \mathrm{ng} / \mathrm{ml}$ of IFN- $\gamma$ (Peprotech, USA) and $100 \mathrm{ng} / \mathrm{ml}$ of LPS 
(Peprotech, USA). Macrophage M2 polarization was obtained by incubation with $20 \mathrm{ng} / \mathrm{ml}$ of IL-4 (Peprotech, USA) and $20 \mathrm{ng} / \mathrm{ml}$ of IL-13 (Peprotech, USA). Macrophages and trophoblasts cocultivation was conducted using the non-contact co-culture transwell system (Corning, USA) for $72 \mathrm{~h}$, in which macrophages were seeded in $0.4 \mu \mathrm{m}$-sized pores inserts and the trophoblast cells were seeded in the 6-well plate.

Recombinant human TGF- $\beta 1$ (Peprotech, USA) was dissolved in $\mathrm{ddH} 2 \mathrm{O}$ and used at a final concentration of $25 \mathrm{ng} /$ mL. LY294002 (PI3K inhibitors) was purchased from Med Chem Express, China. The anti-human neutralizing TGF- $\beta$ antibody was acquired from R\&D Systems, USA.

\section{Overexpression of USP2a}

To generate the USP2a-overexpressing construct, we cloned the coding region sequence of human USP2a into the pLVX-IRESZsGreen vector (Vigene) using the following primers: 5'CGCAAATGGGCGGTAGGCGTG-3' (forward) and 5'CCTCTACAAATGTGGTATGGC-3' (reverse). All constructs were verified by sequencing and transfected into the cells using Lipofectamine 3000 (Invitrogen). Forty-eight hours after transfection, cells were collected for RT-PCR, western blot, migration, and invasion assays.

\section{Knockdown of USP2a}

shRNA was designed explicitly targeting the USP2a, and the sequence was as follows: 5'-GCATGAGGCTCTTTTCACCAA3'. Empty vector or lentivirus packaging shRNA was transfected in HEK293T cells. After 8 hours of transfection, the cell culture medium was changed to a complete medium. After 48 hours, the virus-containing supernatant was collected and filtered with a 0.22 um filter to infect the trophoblast cell line mentioned above. After 24h, Puromycin was added for screening to construct a stably transfected cell line.

\section{Cell Proliferation Assay}

Cell proliferation was assessed using a Cell Counting Kit-8 (CCK-8, Beyotime, Shanghai, China) and EdU assay (Abcam, USA). Trophoblast cells were plated at $2 \times 10^{3}$ cells/well in 96-well plates. Cell proliferation ability was determined at $24,48,72$, or 96 hours using the CCK-8. At the above time point, the supernatant was removed, $10 \%$ CCK- 8 solution was added to each well, and the cells were cultured for two more hours. The enzyme labeling instrument measured the absorbance at $450 \mathrm{~nm}$ wavelength. EdU experiments were carried out to analyze the proliferation of HTR- 8 and BeWo cells according to the manufacturer's instructions. The percentage of EdU positive cells (EdU positive/DAPI positive) was labeled as EdU positive cells. Five technical replicates were done per experiment, and three independent experiments were performed. Colony formation assay was performed as described. After transfection, cells were seeded to six-well plates at densities ranging from 100 to 40,000 cells per well and were put in an incubator for $10-14$ days. After incubation at $37^{\circ} \mathrm{C}$, cells were fixed with glutaraldehyde and stained with crystal violet. Colony formation for each condition was compared with that of untreated control cells. Results were obtained from five replicate wells, and each experiment was repeated three times.

\section{Wound-Healing Assay}

The wound-healing assay was used to evaluate the migration ability of trophoblasts. Cells in the fresh medium were placed into a 6-well plate chamber $\left(5 \times 10^{5}\right.$ cells/well $)$ and incubated for $24 \mathrm{~h}$. When the cells reached $80 \%-90 \%$ confluence, a scratch across the cell surface was made by the plastic pipette tip scraped across the cell surface. Phosphate-buffered saline (PBS) was used to remove floating debris, and the wound was photographed immediately $(0 \mathrm{~h})$. The cells were then cultured in the serum-free medium. The wounds were photographed at $24 \mathrm{~h}$ and $48 \mathrm{~h}$ to measure the extent of wound healing. The sizes of the gaps were measured with Image-Pro Plus software.

\section{Transwell Invasion Assay}

Trypsin Solution (Biosharp, China) was used to collect the transfected trophoblast cells. $5 \times 10^{4}$ cells in $100 \mu \mathrm{l}$ FBS-free medium were seeded into the upper chamber of the transwell insert with $8 \mu \mathrm{m}$ pores (Corning Costar Corp, Corning, NY, USA) which was precoated with matrix Matrigel (BD Biosciences, CA, USA) to evaluate the invasion ability of the trophoblast cells. The transwell chambers without matrix Matrigel were performed to assess the migration ability. The lower chamber was filled by medium supplemented with $10 \%$ FBS $(600 \mu \mathrm{l})$, and the apparatus was incubated at $37^{\circ} \mathrm{C}$. After two days, the cells in the insert were fixed using $4 \%$ paraformaldehyde for thirty minutes and stained using $0.5 \%$ crystal violet. Five random fields under the microscope were used to calculate the number of invaded cells.

\section{Apoptosis Detection Using Annexin V-PE/ 7-AAD Double Staining}

Plasma membrane permeability and phosphatidylserine cell translocation were measured by flow cytometry to define the percentage of apoptotic cells. To this aim, dual staining was performed with PE or APC-conjugated Annexin-V and 7-aminoactinomycin $\mathrm{D}$ (7AAD) using the Annexin-V/7AAD apoptosis detection kit (BD Biosciences). The adherent cells were collected. After washed two times with cold PBS, the cells were resuspended in $100 \mu \mathrm{l}$ PBS. The Cell suspension was co-stained with $10 \mu \mathrm{l}$ 7-AAD and $5 \mu \mathrm{l}$ Annexin V-PE in the dark for 15 minutes at room temperature before analysis using flow cytometry. Early apoptotic cells were defined as Annexin-V+/7AAD- cells and Annexin-V+/7AAD+ as late apoptotic cells. Results were analyzed by flow cytometry (FACS Calibur, BD).

\section{RNA Isolation and RT-PCR}

Total RNA was extracted using the TRIzol RNA purification kit, and NanoDrop 2000 spectrophotometer was used to evaluate RNA purity and quantification. One $\mu \mathrm{g}$ of the total RNA was used to synthesize cDNA using the Takara PrimeScript RT reagent kit according to the manufacturer's instructions. Gene expression was determined by RT-PCR using SYBR Green Supermix (Takara, Dalian, China). A cycle threshold (CT) 
value was obtained for each sample, and duplicate sample values were averaged. The $2^{-\Delta \Delta C T}$ method was used to calculate relative expression of each target gene (25) Briefly, mean CT value of target genes in each sample was normalized to its averaged housekeeping gene CT value to give a $\Delta \mathrm{CT}$ value. This was then normalized to control samples $(\Delta \Delta \mathrm{CT})$, and the $2^{-\Delta \Delta C T}$ value was obtained. The mRNA expression level under a given condition was quantified following normalization to the internal control GAPDH (mean \pm SEM).

\section{Immunohistochemical and Immunofluorescence Staining}

Immunohistochemical staining of human villi or placenta tissue was performed as previously described using rabbit anti-USP2 (dilution 1:200; Abgent, UK), rabbit anti-Cytokeratin 7 (CK7) (dilution 1:200; proteintech), rabbit anti-HLA-G (dilution 1:200; proteintech). Cells were fixed in $4 \%$ paraformaldehyde, permeabilized using 1\% Triton X-100 for $30 \mathrm{~min}$, and blocked with $5 \%$ BSA for one hour at room temperature. Indirect immunofluorescence was performed using primary antibodies, rabbit anti- $\beta$-catenin (1:1,000; Cell Signaling), and secondary antibodies, goat anti-rabbit (1:1,000; proteintech). Nuclear were counterstained with 4',6-diamindino-2-phenylindole (DAPI; Molecular Probes). Images were acquired using an Olympus microscope. The confocal images were acquired by Laser Scanning Biological Microscope (Olympus). Immunohistochemical staining quantifications were assessed using ImageJ software.

\section{Western Blotting and Co-IP Assay}

Western blotting was used to determine protein levels. Protein concentrations were determined using a BCA Protein Assay Kit (Beyotime), according to the manufactures' instructions. SDSPAGE was used to separate the proteins, which were then electrotransferred onto polyvinylidene difluoride (PVDF) membranes (Millipore, USA). After blocking the membranes using $5 \%$ non-fat dry milk in TBST for $1.5 \mathrm{~h}$, the blots were incubated at $4^{\circ} \mathrm{C}$ overnight with various primary rabbit antibodies. The membranes were washed with TBST four times (5 minutes each time) and then incubated with secondary antibodies at room temperature for one hour. The membranes were then washed with TBST four times. Finally, the blots were developed using ChemiDoc ${ }^{\mathrm{TM}}$ Imager from Bio-Rad. The level of GAPDH was used as a loading control to normalize the specific protein levels.

For co-IP, cell extracts were prepared by brief sonication in IP buffer (50 mM Tris-HCl, pH 8.0, $150 \mathrm{mM} \mathrm{NaCl,} 2 \mathrm{mM}$ EDTA, $0.5 \%$ NP-40, $5 \%$ glycerol). The samples were incubated with1$2 \mu \mathrm{g}$ of antibodies overnight, and $30 \mu \mathrm{l}$ of a $25 \%$ protein $A+G$ agarose (ThermoFisher, USA) was added. Three-four hours later, beads were washed with IP buffer three times, and bound proteins were detected by Western blot.

The following primary antibodies were used: anti-USP2 (C-term L523) (Abgent, UK), anti-E-cadherin (Proteintech, Chicago), vimentin (Proteintech, Chicago), Snail (Proteintech, Chicago), and anti-N-cadherin (Proteintech, Chicago). In addition, rabbit polyclonal antibodies, including those recognizing $\mathrm{PI} 3 \mathrm{~K}$, phosphorylated (p)- PI3K, AKT, p-AKT, GSK3 $\beta, \beta$-catenin, BAX, BCL2, and glyceraldehyde 3-phosphate dehydrogenase (GAPDH), were obtained from Cell Signaling Technology (Danvers, MA, USA). The antibodies were used at a working concentration of 1:1000 and were stored at $4^{\circ} \mathrm{C}$. The secondary antibodies were purchased from LI-COR (Lincoln, NE, USA) and used at a dilution ratio of 1:10,000.

\section{Statistical Analysis}

Statistical analyses were performed using SPSS 20 (IBM Corp, USA) and GraphPad Prism 8.0 (GraphPad Software Inc, USA) for Windows. Statistical significance was accepted at a value of $P<0.05$. All experiments were repeated three times. In vitro experiments were analyzed using unpaired Student's $t$-test and ordinary one-way ANOVA test for multiple comparisons.

\section{RESULTS}

\section{The Expression and Localization of USP2a in Placental Villous Tissues}

RT-PCR and Western blot analysis of first-trimester chorionic villous tissue was performed to explore whether USP2a is involved in the pathogenesis of RM. USP2a expression was down-regulated significantly in the villous tissue of patients with RM (Figures 1A, B). Furthermore, immunohistochemical analysis of paraffin-embedded tissue was performed to investigate USP2a localization in the first-trimester villous further. Stronger expression of USP2a was observed in tissue of the NC group, with the positive cells mainly being cytotrophoblasts and EVTs (Figure 1C). To verify the above result, we respectively used immunofluorescence staining with antibodies of CK7 as a marker of cytotrophoblasts, and HLA-G, as a marker of EVTs on human villous tissue, as shown in Figure 1D. Immunofluorescence shows resemblant results. The above results demonstrated the aberrant expression of USP2a might be involved in the pathogenesis of RM.

\section{The Influences of USP2a on the Proliferation and Apoptosis Ability of Trophoblast Cells}

To gain a deeper insight into the molecular function of USP2a, we first detected the mRNA expression of USP $2 \mathrm{a}$ in the trophoblast cell lines, including HTR-8/SVneo, BeWo, JEG-3, and JAR. As shown in Figure 2A, we found that the relative level of USP2a in BeWo and HTR- 8 cell lines was relatively higher and lower than those in the other two cell lines. Thereby, we chose these two cell lines for subsequent exploration. sh-USP2a significantly decreased the expression of USP2a in BeWo, while p-USP2a had the opposite effect in HTR8 (Figure 2B). Following this, EdU labeling, CCK-8 analysis, and colony formation were performed to investigate the role of USP2a in trophoblasts proliferation. EdU assay revealed that knockdown of USP2a reduces the percentage of EdU-positive cells. Meanwhile, EdU positive cells increased due to USP2a overexpression (Figure 2C). Comparable results of that higher USP2a level could induce trophoblasts proliferation were obtained 


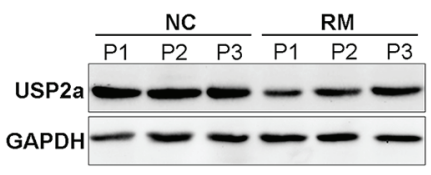

C
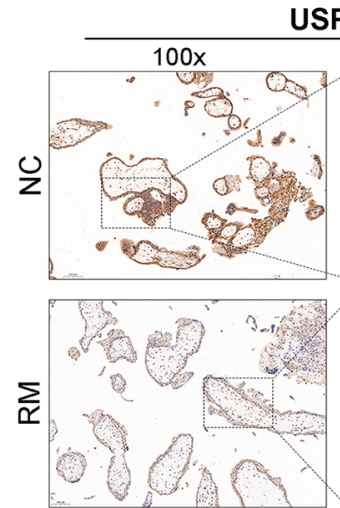

D
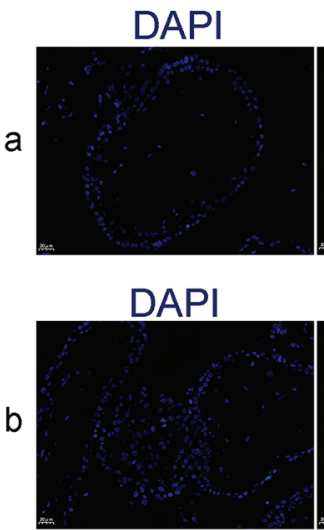

USP2a
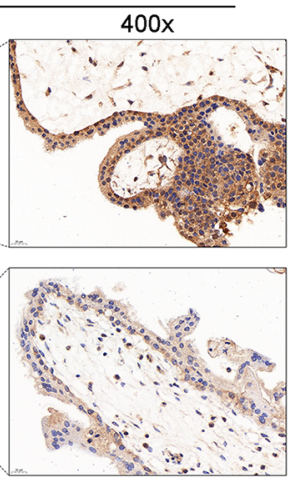
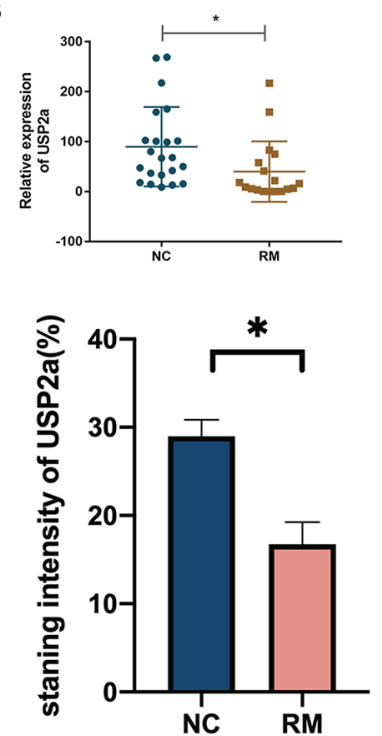
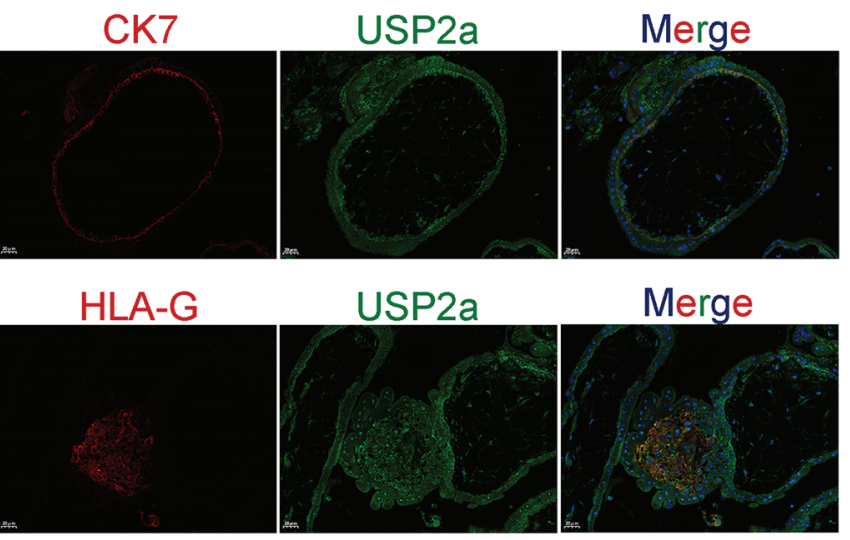

Merge

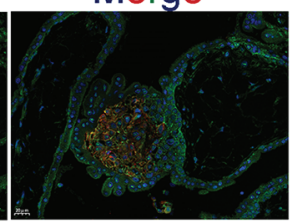

FIGURE 1 | USP2a is down-regulated in placental in patients with recurrent miscarriage (RM). (A, B) Comparison of relative mRNA and protein expression levels of USP2a in placental villous tissues between RM patients $(n=18)$ and normal women $(n=22)$ by RT-PCR and western blot. (C) Representative immunohistochemical images of villous expression and localization of USP2a in RM patients ( $n=22)$ and normal women $(n=25)$. The staining intensity was quantified using the Image software. Graphs show the means \pm SD. Magnification, 100x and 400x. (D) Representative immunofluorescence images: (a) double immune-staining of CK7 (red) and USP2a (green). Magnification, 400x; (b) double immune-staining of HLA-G (red) and USP2a (green) Magnification, 400x. ${ }^{\star} P<0.05$. Scale bar = 100 $\mu$ m (100x); Scale bar $=20 \mu \mathrm{m}(400 \times)$. mRNA, messenger RNA; RT-PCR, quantitative real-time polymerase chain reaction; RM, recurrent miscarriage; USP2a, ubiquitin-specific protease 2a; CK7, cytokeratin 7; HLA-G, human leukocyte antigen G.

from the CCK-8 assay (Figure 2D) and the colony formation assay (Figure 2E). Apoptosis experiments indicated that knockdown of USP2a promotes apoptosis (Figure 2F). We also observed decreased level of anti-apoptotic protein BCL2 in trophoblast cells when USP2a was knockdown, whereas the abundance of the proapoptotic protein BAX increased obviously. Opposite results were obtained in the USP2a overexpression cell line (Figure 3D).

\section{USP2a Regulates the Invasion and Migration Ability of Trophoblast Cells}

Next, the invasion and migration capacities of BeWo and HTR8 were estimated. Knockdown of USP2a inhibits trophoblast cell migration in imaging assay after the scratch test. In contrast, overexpression of USP2a exhibited a faster closure of the wound when compared to the control group (Figure 3A). When USP2a was knocked down, the numbers of invading cells were significantly decreased, whereas the numbers of invading cells were considerably raised when USP2a was overexpressed (Figures 3B, C). The results showed that knockdown of USP2a significantly inhibited trophoblasts migration and invasion compared with the control group, whereas overexpression of USP2a had an opposite effect. Considering the previous evidence, it seems that trophoblasts with increased migration and invasion capacity usually experience EMT (26). We analyzed several EMT markers by western blot (Figure 3D). As expected, the overexpression of USP2a in trophoblasts induced an increase in the EMT phenotype. The knockdown of USP2a significantly 
A
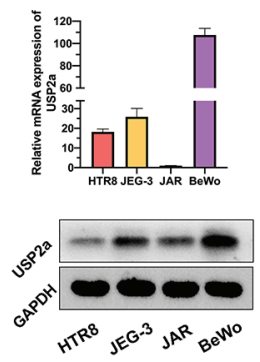

C

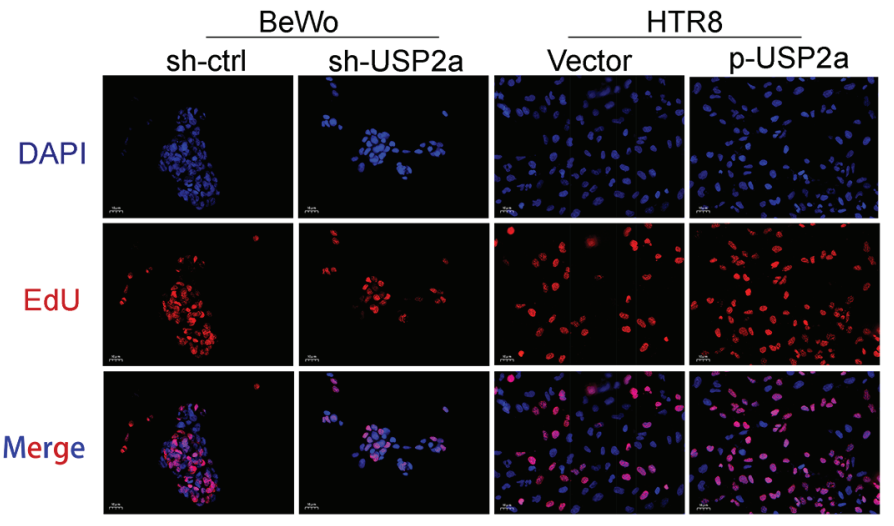

D

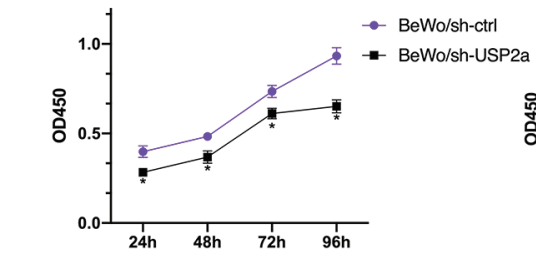

E

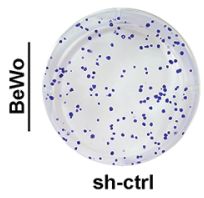

$\mathbf{F}$

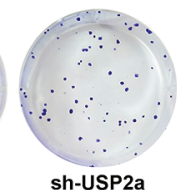

BeWo

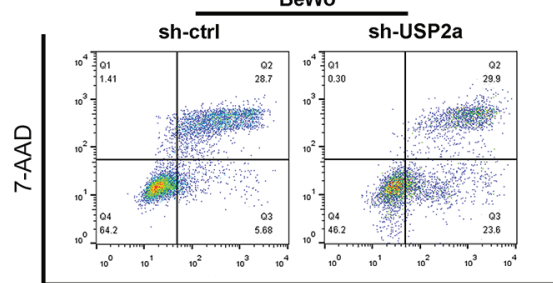

B

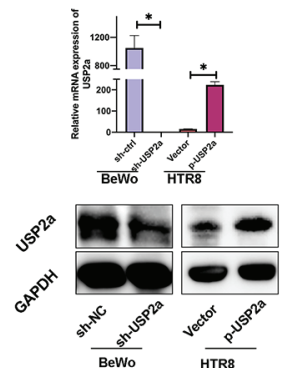

HTR8
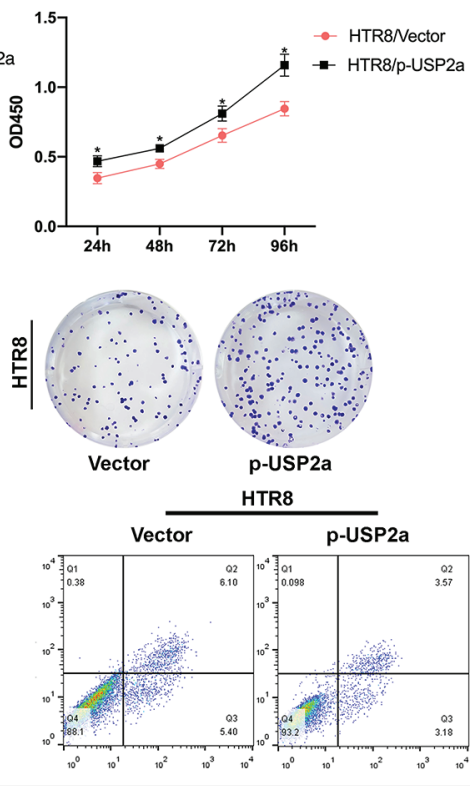

Annexin V/APC

FIGURE 2 | USP2a promoted human trophoblast-derived cell lines proliferation and inhibited cell apoptosis in vitro. (A) Relative USP2a mRNA and protein expression levels in four human trophoblast-derived cell lines were measured by RT-PCR and western blot, respectively. GAPDH was used as the loading control. (B) Knockdown of USP2a by shRNA (sh-USP2a) and upregulation of USP2a by plasmid expression were conducted in the BeWo and HTR8 cell lines. The mRNA expression and protein level of USP2a were confirmed by RT-PCR and western blot. (C) Representative images of EdU labeling assay in p-USP2a/HTR8 and shUSP2a/BeWo. DAPI staining blue; EdU staining red. Scale bar $=10 \mu \mathrm{m}$ (D) Cell proliferation was measured 48 hours after transfection using the CCK8 assay. (E) Knocking down USP2a inhibits cell clone formation significantly in BeWo cells. Overexpression of USP2a promotes cell clone formation in HTR8 cells. (F) Quantitative flow cytometry measurements of apoptosis in trophoblast cell lines after transfection of either p-USP2a or shRNA. The percentage of live (7AAD-/Annexin $\mathrm{V}-$ ), early apoptotic (7AAD-/Annexin $\mathrm{V}+$ ), and dead (7AAD+/Annexin $\mathrm{V}+$ ) cells was determined. ${ }^{*} P<0.05$. 

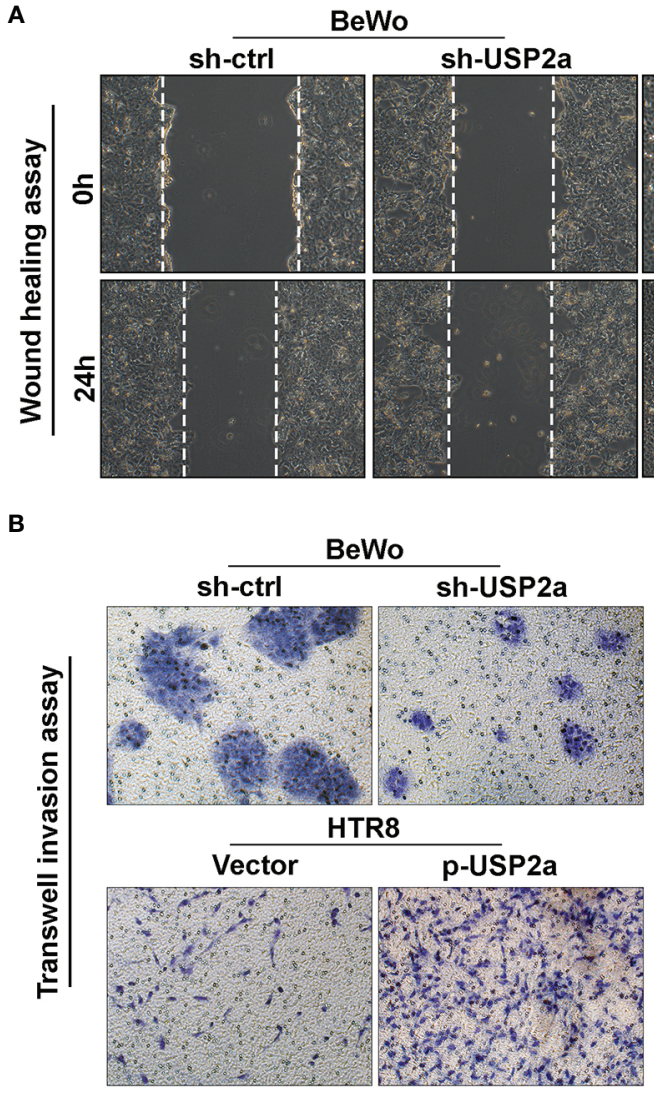

D

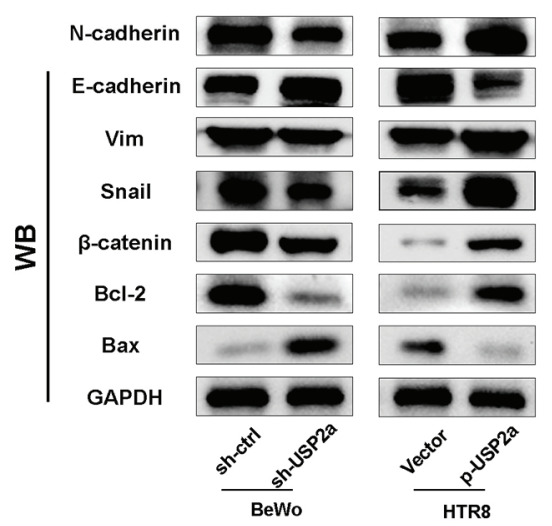

HTR8

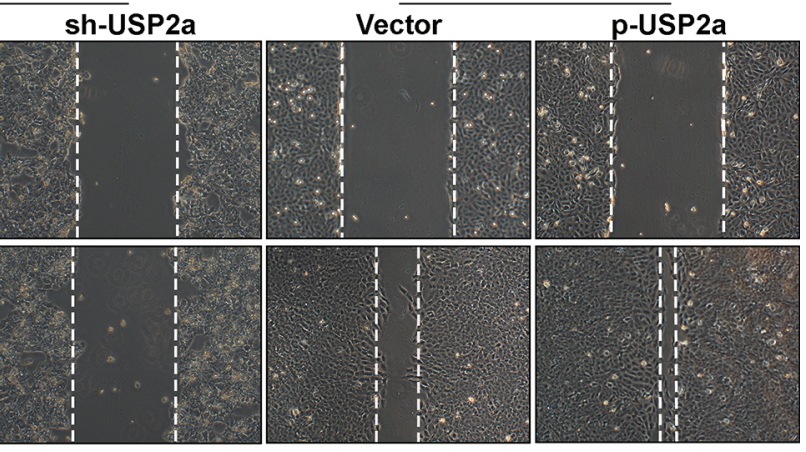

C
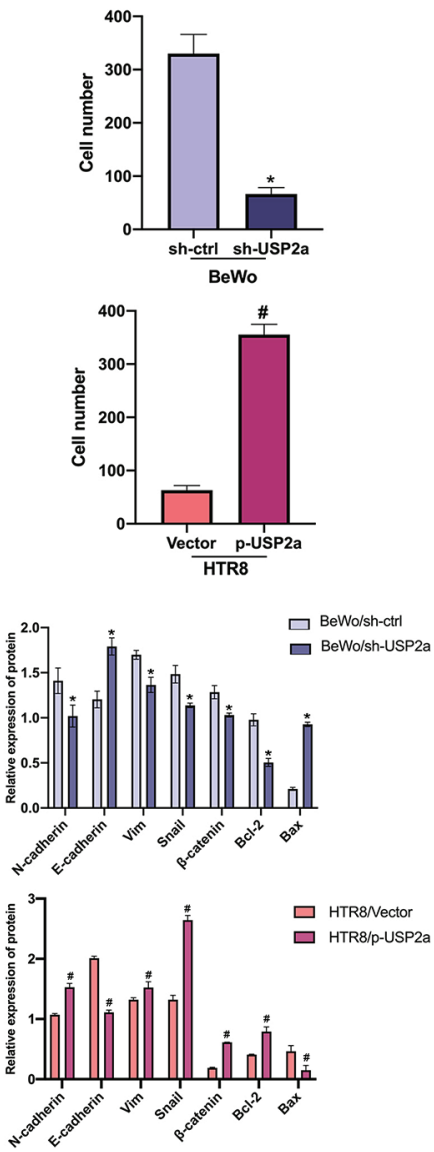

FIGURE 3 | Effects of USP2a on the invasion and migration of trophoblast cells using the Transwell invasion assay and the wound-healing assay. (A) Wound healing scratches were imaged immediately and $24 \mathrm{~h}$ after initial scratch time to quantify relative migration. (B) After transfection, BeWo or HTR8 cells (in serum-free medium) were seeded into inserts and incubated for $48 \mathrm{~h}$ for invasion assay. Cells at the lower surface of the membrane were counted and analyzed under a light microscope in five random fields. The staining intensity was quantified using the ImageJ software. Graphs show the means \pm SD. (C) Western blot Graphs show the mean \pm SD. Quantitative analysis of the average invasive cell numbers in each group. (D) The levels of E-cadherin, $\mathrm{N}$-cadherin, Vimentin, Snail, $\beta$-catenin, Bax, and Bcl2 were measured using western blotting and quantitative analysis of the proteins was performed. ${ }^{\star} P<0.05$ vs. sh-Ctrl ${ }^{\#} P<0.05$ vs control vector, original magnification: $\times 100$ (A) $\times 200$ (B).

inhibited EMT, as shown by the decreased expression of mesenchymal marker N-cadherin, Snail, and Vimentin and increased expression of epithelial marker E-cadherin, where overexpression of USP2a resulted in the opposite effects.
Besides, we also observed an enhanced expression of $\beta$-catenin in USP2a-overexpression trophoblasts. Altogether, these results demonstrated that USP2a could regulate the invasion and migration ability of trophoblasts. 


\section{Reduced USP2a Expression Impairs Nucleocytoplasmic Translocation of $\beta$-Catenin and Decreases Trophoblasts Invasion}

$\beta$-catenin combines with the intracellular segment of E-cadherin to form a cadherin-catenin complex so that E-cadherin is located at the junction between cells maintaining cell adhesion $(27,28)$. If $\beta$-catenin accumulates in the cytoplasm, it will enter the nucleus from the cytoplasm and undergo nuclear translocation if the concentration reaches a certain level. In that case, $\beta$-catenin into the nucleus can activate the expression of downstream target genes, thereby promoting EMT (27). We used IF to detect the location and expression of $\beta$-catenin in HTR8 cells overexpressing USP2a and BeWo cells with USP2a knockdown. The results showed that the overexpression of USP2a significantly increased the expression level of $\beta$-catenin, especially the nuclear localization of $\beta$-catenin, while the knockdown of USP2a showed the opposite result (Figure 4A). Then we further detected the expression of $\beta$ catenin in the cytoplasm and nucleus by Western blot, and the results were consistent with the IF results (Figure 4B). we also detected the cooperation of USP2a and $\beta$-catenin in trophoblast cells by co-IP (Figure $4 \mathrm{C}$ ). Since the degradation of $\beta$-catenin is regulated by glycogen synthase kinase- $3 \beta$ (GSK- $3 \beta$ ), and GSK-3 3 is a downstream molecule of the PI3K/Akt signaling pathway (29).
Therefore, we suspect that USP2a may regulate EMT through the PI3K/Akt/GSK-3 $\beta$ signaling pathway. We detected the expression of the pathway proteins mentioned above when USP2a is overexpressed or downregulated (Figure 5A). This confirmed our suspicions. p-USP2a/HTR8cells were treated with PI3K inhibitor LY294002, and then the changes of EMT-related indicators were detected by Western blot. We found that after LY294002 treatment, E-cadherin expression increased considerably, while the expression of N-cadherin, Vimentin, and Snail decreased significantly (Figure 5B). Likewise, the invasion and migration ability were reduced to a degree in the p-USP2a/HTR8 cells (Figure 5C).

\section{M2 Macrophage-Secreted TGF- $\beta$ Cooperates With USP2a in Trophoblasts to Promote Invasion}

Previous studies reported that abnormal levels of various cytokines, including IL-4, IL- 6 , and IFN- $\gamma$ regulated trophoblast growth and invasion in patients with RM (30). We wondered whether these factors also are involved in the regulation of USP2a expression in trophoblasts and work in concert to play a role in the pathology of RM. We stimulated HTR8 cells with different cytokines (IFN- $\gamma$, IL-13, IL-4, IL-6, LPS, or TGF- $\beta$ ) and then detected the relative expression levels of USP2a mRNA and protein by RT-PCR and Western blot. The
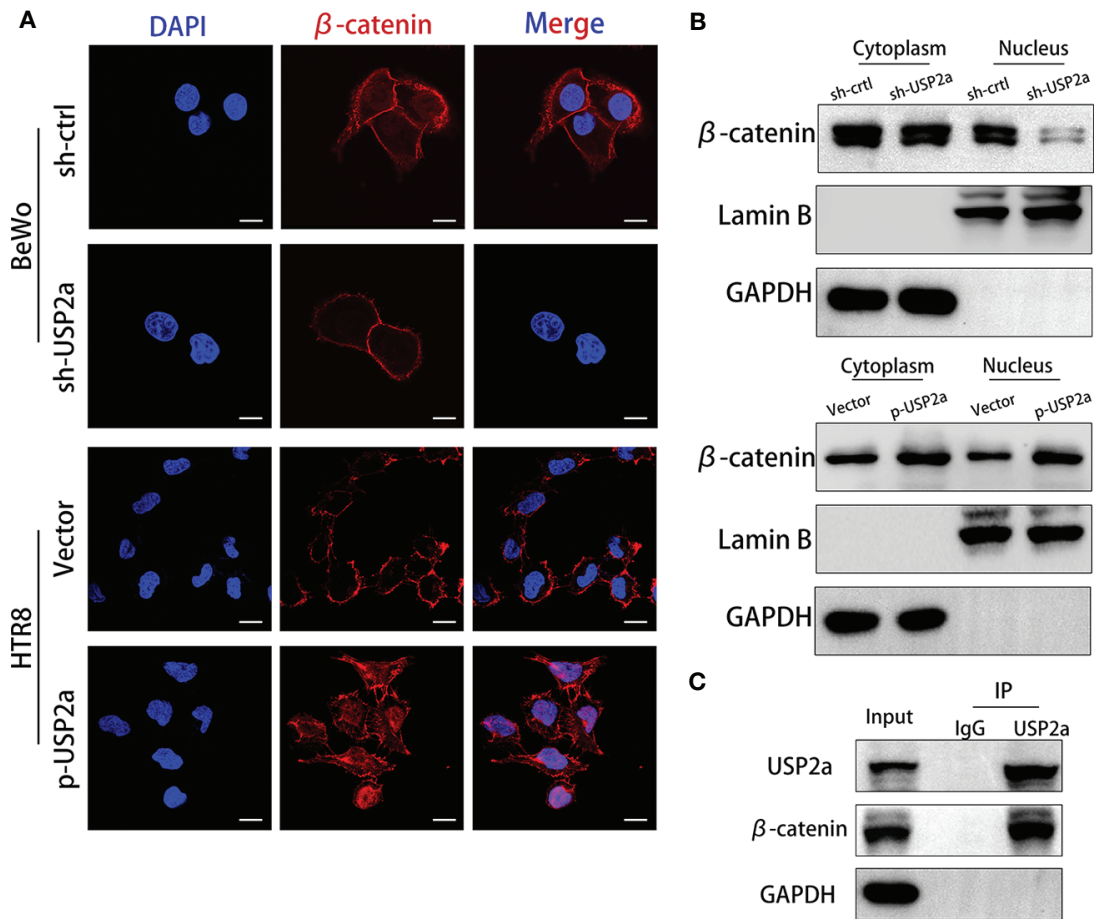

FIGURE 4 | Reduced USP2a Expression impairs nucleocytoplasmic translocation of $\beta$-catenin. Knockdown of USP2a attenuates nuclear translocation of $\beta$-catenin in BeWo cells. Overexpression of USP2a induces nuclear translocation of $\beta$-catenin in HTR8 cells BeWo cells were transfected with sh-Ctrl or sh-USP2a, and HTR8 cells were transfected with p-USP2a or vector. Both cells were cultured for 48 hours. (A) The cells were seeded to immunofluorescent staining for $\beta$-catenin (red) and counterstained with DAPI (blue) to assess the subcellular localization of $\beta$-catenin and (B) western blot was performed to compare the levels of $\beta$-catenin in the nucleus and cytoplasm. GAPDH and lamin B were used as the loading control. (C) Co-IP assays of the interaction between USP2a and $\beta$-catenin in HTR8. Scale bar $=10 \mu \mathrm{m}$. 


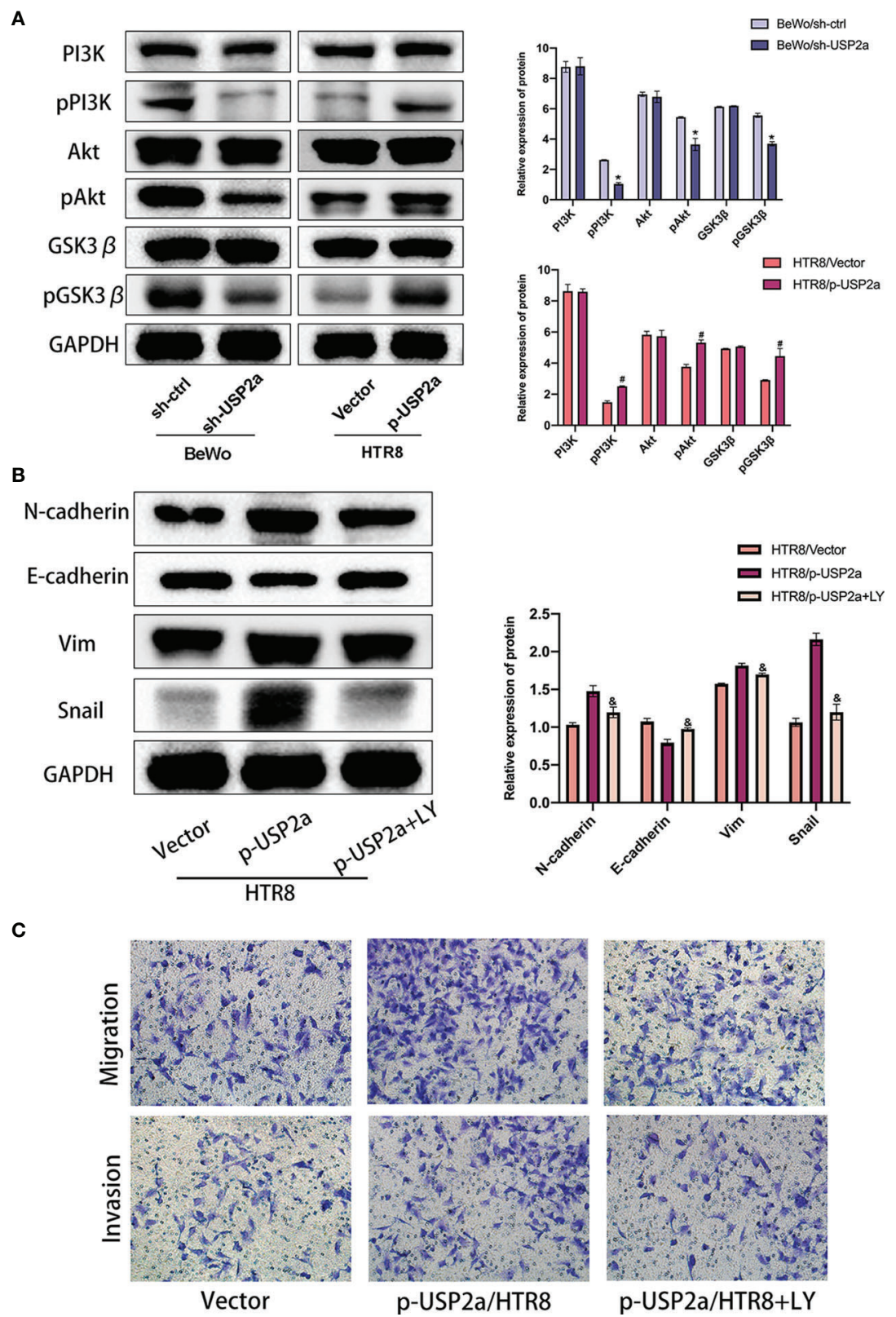

FIGURE 5 | USP2a promotes EMT, migration and invasion of trophoblasts via activating PI3K/Akt/GSK3 $\beta$ pathway. (A) Western blot analyzes relative protein level of sh-Ctrl/BeWo, sh-USP2a/BeWo, Vector/HTR8, p-USP2a/HTR8. (B) Western blot analysis of sh-Ctr//BeWo, sh-USP2a/BeWo, Vector/HTR-8, p-USP2a/HTR8 in the

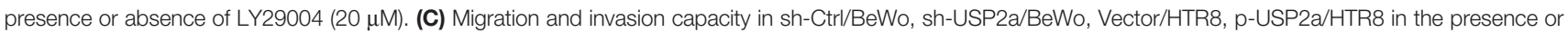
absence of LY294002 $(20 \mu \mathrm{M})$ was determined by transwell system. Representative photographs of migratory and invasive cells (magnification, $\times 200)$ are shown. ${ }^{*} P<0.05$, vs. sh-Ctrl ${ }^{\#} P<0.05$ vs vector. ${ }^{\circledR} P<0.05$ vs $p-U S P 2 a / H T R 8$.

results showed that the expression of USP2a increased significantly after IL- 6 and TGF- $\beta$ stimulation, but there was no significant change after IFN- $\gamma$, IL-13, IL-4, and LPS stimulation (Figure 6A). Since our group and others have confirmed the effect of IL- 6 on trophoblasts in the early stage of pregnancy $(16,30)$, we will mainly explore the effect of TGF- $\beta$. We also detected TGF- $\beta$ expression in trophoblast cell lines that were transfected with a USP2a-overexpressing plasmid or control vector. No significant change was observed in TGF- $\beta$ expression in USP2a-expressing cells in RT-PCR analysis. Next, we detected the TGF- $\beta$ receptor (TGFBR1) on trophoblasts in the placental villous tissues and found the colocalization of TGFBR1 and USP2a by co-immunofluorescence staining (Figure 6B). Therefore, we postulated that USP2a affects trophoblasts invasion by interacting with TGFBR1. To test this assumption, we conducted co-IP experiments. Co-IP 


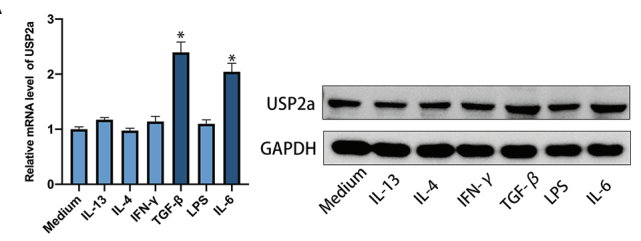

B

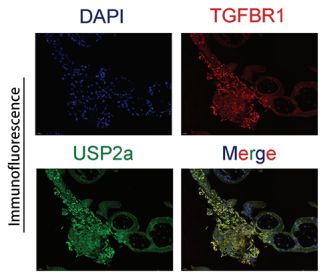

C

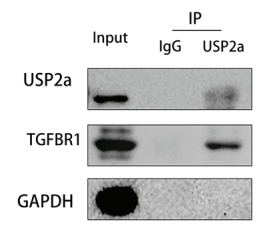

D
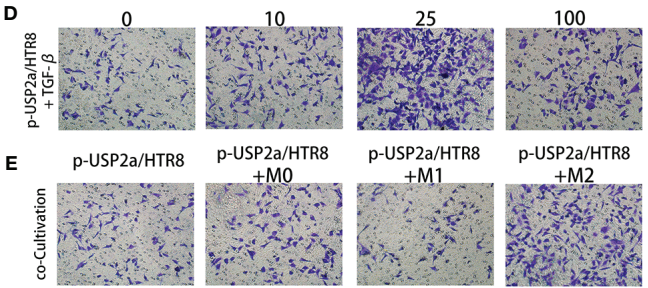

$\mathrm{p}-\mathrm{USP2} 2 \mathrm{a} / \mathrm{HTR} 8$

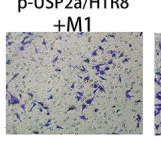

$\mathrm{p}-\mathrm{USP} 2 \mathrm{a} / \mathrm{HTR} 8$
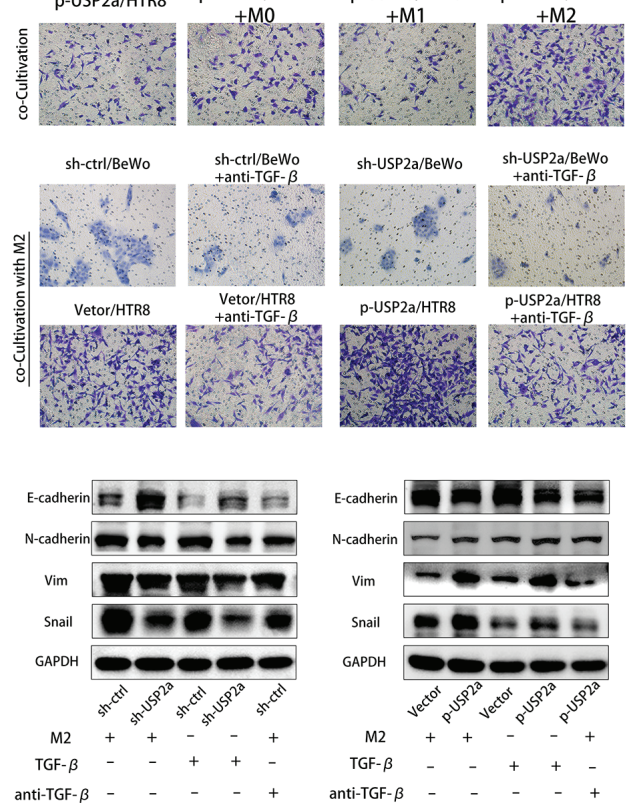

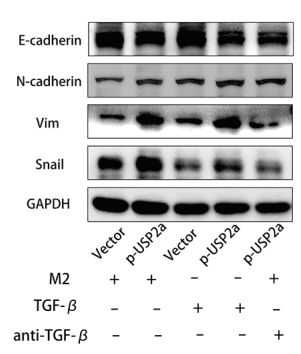

FIGURE 6 | M2 Macrophage-secreted TGF- $\beta$ cooperate with USP2a in trophoblasts to promote invasion. (A) Western blot and quantitative RT-PCR analysis of USP2a expression in HTR8 treated with IFN- $\gamma$, IL-13, IL-4, IL-6, LPS, or TGF- $\beta$ for 24 hours. (B) Immunostaining for TGFBR1 and USP2a in villous from normal pregnancy woman (Scale bar $=20 \mu \mathrm{m})$. (C) co-IP assays of the interaction between USP2a and TGFBR1 in p-USP2a/HTR8. (D) Cell invasion capacity in HTR8 alone, HTR8 with $10 \mathrm{ng} / \mathrm{ml}$, 25ng/ml, or $100 \mathrm{ng} / \mathrm{ml}$ rhTGF- $\beta$ by transwell system. (E) Cell invasion capacity in $\mathrm{p}-\mathrm{USP} 2 \mathrm{a} / \mathrm{HTR} 8$ alone, $\mathrm{p}-\mathrm{USP} 2 \mathrm{a} / \mathrm{HTR} 8 \mathrm{co}$-cultured with M0 macrophage, co-cultured with M1 macrophage, and co-cultured with M2 macrophage by transwell system. (F) Cell invasion capacity in sh-Ctrl/BeWo, sh-USP2a/BeWo, Vector/HTR8, p-USP2a/HTR8 co-cultured with M2 macrophage or above cells co-cultured with TGF- $\beta$ depleted M2 macrophage were determined by transwell system, respectively. (G) Western blot assay of EMT proteins in sh-Ctrl/BeWo, shUSP2a/BeWo, Vector/HTR8, p-USP2a/HTR8 co-cultured with M2 macrophage or above cells co-cultured with TGF- $\beta$ depleted M2 macrophage were determined by transwell system, respectively. ${ }^{\star} P<0.05$, vs. HTR8. DAPI staining blue; TGFBR1 staining red; USP2a staining green. Representative photographs of invasive cells (magnification, $\times 200$ ) are shown.

experiments further corroborated the interaction between USP2a and TGFBR1 (Figure 6C). We check the invasion of p-USP2a/ HTR8 treated with exogenous rhTGF- $\beta$. At a concentration of $25 \mathrm{ng} / \mathrm{ml}$ of TGF- $\beta$, p-USP2a/HTR8 exhibited the most invasion ability (Figure 6D). TGF- $\beta$ is one of the important cytokines produced by M2 phenotype macrophages in the fetal-maternal interface (13). p-USP2a/HTR8 were co-cultivated with M0, M1, and M2 macrophages separately. Compared with co-cultivation with M0 macrophages, co-cultivation with M2 macrophages significantly increased trophoblast invasion (Figure 6E). This effect is more evident in the USP2a-upregulating HTR8 cells. Intriguingly, when neutralizing antibody for TGF- $\beta$ was added, the invasion ability of trophoblasts promoted by M2 was alleviated. A similar effect was confirmed in USP2adownregulated BeWo cells (Figure 6F). The relative expression evaluated by western blotting showed similar effects (Figure 6G). These results strongly suggested that M2 macrophage-secreted TGF- $\beta$ may work together with USP $2 a$ as one of the regulators of the biological functions of trophoblasts in patients with RM.

\section{DISCUSSION}

It is generally recognized that placental insufficiency is one of the main culprits in RM arising from shallow trophoblast invasion at the initial stages of pregnancy $(31,32)$. The present study illustrated that USP2a is low-expressed in the placental villous samples of RM women, and aberrant expression of USP2a can impact trophoblast invasion by regulating the EMT process. Further research showed that USP2a regulates $\beta$-catenin translocating into the nucleus through PI3K/AKT/GSK3 $\beta$ pathway, thereby inducing trophoblasts migration and invasion. Moreover, TGF- $\beta$ secreted by M2 macrophages could interact with USP2a in trophoblasts to regulate placentation.

Ubiquitination describes the process during which the 76residue protein termed ubiquitin is covalently conjugated to intercellular proteins (17). Ubiquitination is reversible and precisely regulated due to the activity of deubiquitinating enzymes (18). Together these two systems collaborate as control of cell signaling networks by regulation of protein interactions and activities. USP2a, a cysteine protease, as a member of the ubiquitination specific protease family, is extensively expressed in many human cancers and often correlates with tumor progression and poor prognosis. Previous studies suggest USP2a interacts with FAS which often overexpresses in biologically aggressive human tumors.

Further, functional inactivation of USP2a results in decreased FAS protein with reinforced apoptosis of prostate cancer cells (33). Also, Priolo et al. report that USP2a overexpression in prostate cancer contributes to tumorigenesis by repressing p53 (34). Allende-Vega further illustrated this mechanism that ectopic expression of USP2a causes accumulation of Mdm2 and promotes Mdm2-mediated p53 degradation (35). USP2a could promote hepatocellular carcinoma invasion via deubiquitinating RAB1A (36). These studies indicated USP2a presented oncogenic properties $(34,37,38)$. Since the processes of trophoblast proliferation, migration, invasion, and apoptosis 
at the implantation site share similar biochemical mediators of malignant tumor metastasis (39). Therefore, we examined the role of USP2a in placentation during early pregnancy. The results of our study indicate USP2a could promote trophoblast invasion, migration, and proliferation as well as reduce apoptosis. Thus, we illustrate the critical role of USP2a in the pathogenesis of RM by affecting the invasion and migration of trophoblasts.

We also observed that overexpression of USP2a resulted in upregulation of $\beta$-catenin and downregulation in E-cadherin. E-cadherin/ $\beta$-catenin complex located in cell-cell adherent junctions in the cell membrane creates tight cell-cell interactions, thus restraining cell mobility and maintaining epithelial integrity $(27,28)$. However, decreased E-cadherin expression results in degradation of the E-cadherin/ $\beta$-catenin conjunction. Therefore, $\beta$-catenin is released into the cytoplasm of trophoblasts, and next translocated from the cytosol into the nucleus and induces EMT genes transcription (40-42). Accordingly, we evaluated the level of $\beta$-catenin nuclear translocation. The overexpression of USP 2 a promoted the nuclear transfer of $\beta$-catenin in trophoblast cells. Instead, the knockdown of USP2a suppressed this process. Unsurprisingly the trophoblasts acquired mesenchymal-cell markers prone to migration and invasion, such as $\mathrm{N}$-cadherin, vimentin, and Snail. This is consistent with previous literature that the moderate $\beta$-catenin signaling pathway promotes the invasion of EVTs and provides the basis for successful embryo implantation (43). Our results also suggest that USP 2 a interreact with $\beta$-catenin. As USP2a has been reported to deubiquitinate and stabilize to promote $\beta$-catenin nuclear accumulation and transcriptional activity of $\beta$-catenin (24). We believe USP2a could enhance trophoblast invasion by both PI3K/Akt/GSK3 $\beta / \beta$-catenin pathways and deubiquitinating $\beta$-catenin.

The regulation of the immune system during pregnancy is a fascinating and complex mechanism. Interactions between the maternal stromal components and fetal-derived trophoblasts of the uterus and placenta are not an isolated occurrence but rather are under a superimposition layer of regulation by the maternal immune cells that populate the decidua (12). On the one hand, embryo implantation requires a local inflammatory environment that promotes cell clearance, angiogenesis, and cell growth (9). On the other hand, the fetus as a semi-allograft that carries paternal antigen would be rejected if there were no tolerance environment of the maternal immune system during pregnancy (44). Macrophages represent the second most leukocyte subsets in the human maternal-fetal interface. The polarization of decidual macrophages is biased to the M1 phenotype during the pre-implantation period. Then they begin to transition to a mixed M1/M2 profile when the trophoblasts establish attachment into the endometrium and invade the uterine stroma. In general, decidual macrophages are characterized by an immunosuppressive phenotype as $\mathrm{M} 2$ polarization secreting IL-10, TGF- $\beta$, and G-CSF, supporting fetal-maternal immune tolerance during implantation $(45,46)$. Abnormal macrophage polarization is related to many pregnancy complications, such as PE, premature birth, and RM (47). The previous study has shown that TGF- $\beta$ can increase the invasiveness of placental cells in rats
(48). In our study, TGF- $\beta$ promoted trophoblast invasion in a dose-dependent manner. At a concentration of $25 \mathrm{ng} / \mathrm{ml}$ of TGF- $\beta$, trophoblast cells exhibited the most invasion ability, while $100 \mathrm{ng} / \mathrm{ml}$ of TGF- $\beta$ shows little pro-invasive function in trophoblast. A previous study revealed the expression of TGF- $\beta$ in pregnant rats is increased following implantation (days 5.5 to 6.5) and is maximal during regression of the decidual basil (day 14) (49). These results corroborate the findings of previous work that shows TGF- $\beta$ can also restrict trophoblasts invasion in late pregnancy to prevent preeclampsia (50). We assume that the regulation of TGF- $\beta$ on trophoblast is under complicated mechanisms through the whole pregnancy period. Here we illustrated USP2a collaborates with TGF- $\beta$ to promote trophoblast cell invasion to help maintain normal pregnancy. Our results also demonstrate USP2a could interreact with TGFBR1. Therefore, we speculated that during normal pregnancy, decidua macrophages present an M2 phenotype and secrete TGF- $\beta$ as crosstalk between decidua macrophages and the trophoblast cells. Meanwhile, TGF- $\beta$ could promote the USP2a expression of trophoblast cells. USP2a could further interreact with TGFBR1, thus improving trophoblast invasion ability (Figure 7). However, the exact mechanism remains unclear and needs further research.

Chaiworapongsa reported a case that a 38-year-old RM patient who took the histopathological examination of the placenta from a previous pregnancy showed fibrinoid deposition in the intervillous space surrounding more than $50 \%$ of the villi in some full-thickness sections. After receiving statin therapy, the patient delivered a neonate without major complications vaginally at 34 weeks of gestation (51). An arraybased kinase assay spanning the entire human kinome indicated statins induce upregulation of PTEN activity leading to downregulation of the PI3K/Akt signaling (52). The PI3K/Akt signaling pathway is one of the major driving forces in a range of cellular functions and dysregulation of PI3K/Akt has been implicated in many human diseases including RM (53). Interestingly, we also detected USP2a expression in the glandular epithelium and stromal cells of human endometrial tissue and decidua tissue. When human endometrial stromal cells were cultured and stimulated by progesterone and cAMP, levels of USP2a were increased. In comparison, decidualization was suppressed markedly silencing USP2a. This suggests that progesterone and cAMP may modulate USP2a production and that USP2a may contribute to regulating decidualization. Together we can assume that USP2a plays an essential role in early pregnancy.

Several limitations need to be pointed out regarding the current study. First, whether USP2a has an impact on regulating macrophage polarization still requires further research. Second, in this study, we exclusively focused attention on the effects of macrophages but ignored other immune cells. Numerous immune cells, cytokines, chemokines, and signal molecules from the maternal-fetal interface microenvironment work synergistically to establish and maintain pregnancy. Finally, the findings from this study came from the clinical tissue and in vitro verification, which needs 


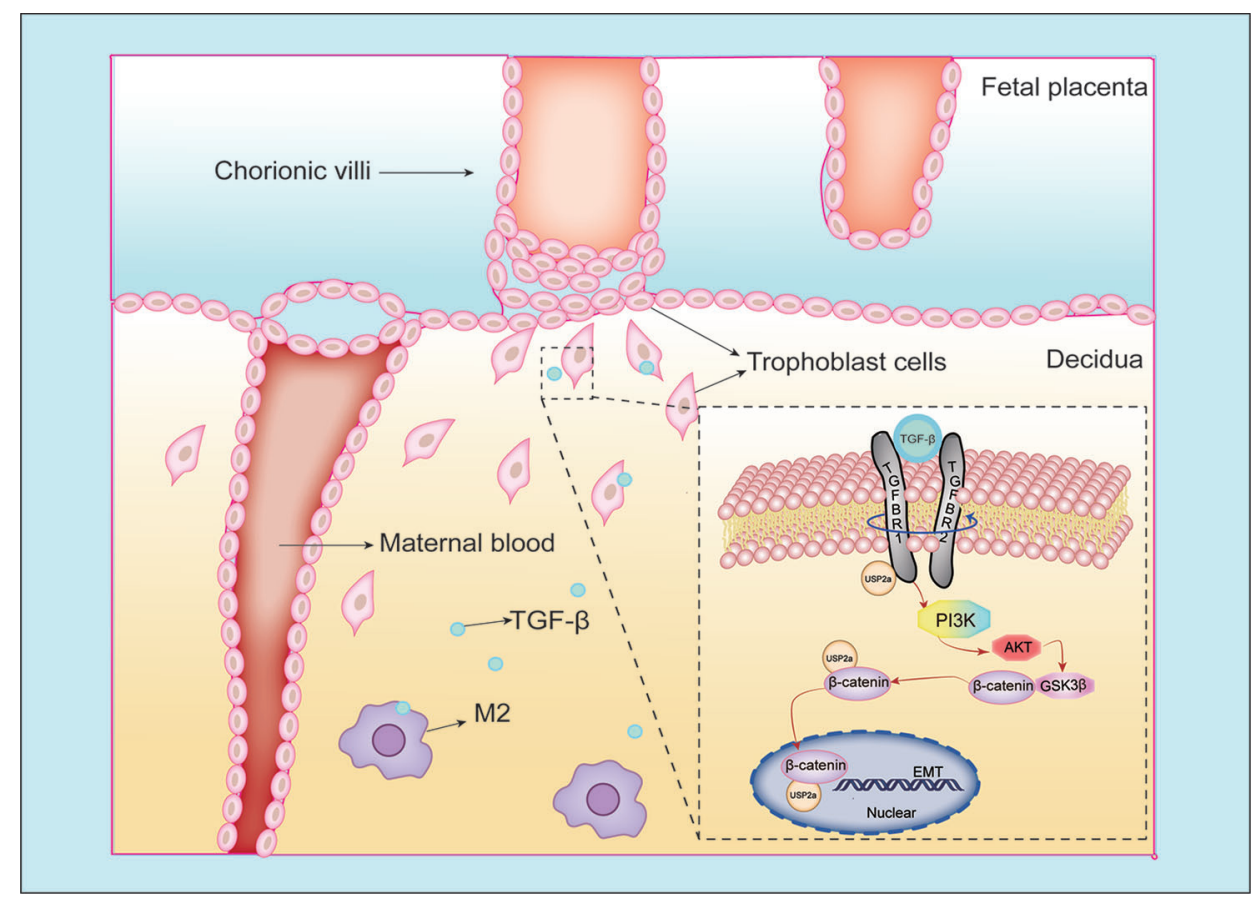

FIGURE 7 | Schematic diagram of the interaction between trophoblasts and macrophages at the maternal-fetal interface. Our study illustrated USP2a facilitates the

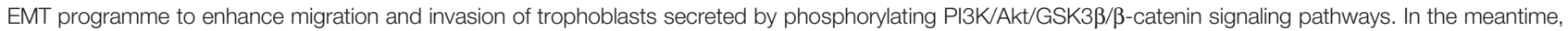
USP2a could interreact with $\beta$-catenin as a "shortcut" to promote nuclear $\beta$-catenin accumulation. TGF- $\beta$ secreted by M2 macrophage could work together with USP2a to promote trophoblast invasion. Thereby, USP2a participates in regulating the establishment and maintenance of normal pregnancy.

further identification on in vivo models. These limitations refer that these findings of the present study need to be interpreted cautiously.

In summary, this study provides new insights into the distinctive trophoblast proliferation and invasion events during the early stage of human pregnancy. Furthermore, the evidence from this study suggests transplacental regulation of cellular immunity and a possible regulating mechanism of TGF- $\beta$ secreted by macrophages in regulating trophoblast invasion. These novel findings have important implications for the role of trophoblast invasion in maintaining a successful pregnancy.

\section{DATA AVAILABILITY STATEMENT}

The original contributions presented in the study are included in the article/supplementary material. Further inquiries can be directed to the corresponding authors.

\section{ETHICS STATEMENT}

The studies involving human participants were reviewed and approved by The Ethics Committee of Renmin Hospital of Wuhan University. The patients/participants provided their written informed consent to participate in this study.

\section{AUTHOR CONTRIBUTIONS}

Conceptualization, JW and JD. Data curation, JW. Formal analysis, SZ. Investigation, JW, XC, and SZ. Methodology, JD. Project administration, JW and SY. Supervision, JD. Validation, $\mathrm{XC}$ and JD. Visualization, JD and SY. Writing - original draft, JW. Writing - review and editing, TY and YZ. All authors contributed to the article and approved the submitted version.

\section{FUNDING}

This work was supported by the following grants: National Key Research and Development Program of China (No. 2018YFC1004601), the National Natural Science Foundation of China (No. 81801540, 81771662) and the Fundamental Research Funds for the Central Universities (2042021kf0082)

\section{ACKNOWLEDGMENTS}

The authors thank Mrs. Qiong Ding from the Central Laboratory of Renmin Hospital of Wuhan University for her laboratory assistance, help, advice, and support. 


\section{REFERENCES}

1. Rai R, Regan L. Recurrent Miscarriage. Lancet (2006) 368(9535):601-11. doi: 10.1016/S0140-6736(06)69204-0

2. Popescu F, Jaslow CR, Kutteh WH. Recurrent Pregnancy Loss Evaluation Combined With 24-Chromosome Microarray of Miscarriage Tissue Provides a Probable or Definite Cause of Pregnancy Loss in Over $90 \%$ of Patients. Hum Reprod (2018) 33(4):579-87. doi: 10.1093/humrep/dey021

3. Arck PC, Hecher K. Fetomaternal Immune Cross-Talk and Its Consequences for Maternal and Offspring's Health. Nat Med (2013) 19(5):548-56. doi: 10.1038/nm.3160

4. Mayhew TM. Turnover of Human Villous Trophoblast in Normal Pregnancy: What do We Know and What do We Need to Know? Placenta (2014) 35 (4):229-40. doi: 10.1016/j.placenta.2014.01.011

5. Nieto MA. Epithelial-Mesenchymal Transitions in Development and Disease: Old Views and New Perspectives. Int J Dev Biol (2009) 53(8-10):1541-7. doi: $10.1387 /$ ijdb.072410mn

6. J ED, Pollheimer J, Yong HE, Kokkinos MI, Kalionis B, Knöfler M, et al. EpithelialMesenchymal Transition During Extravillous Trophoblast Differentiation. Cell Adh Migr (2016) 10(3):310-21. doi: 10.1080/19336918.2016.1170258

7. Huppertz B. Traditional and New Routes of Trophoblast Invasion and Their Implications for Pregnancy Diseases. Int J Mol Sci (2019) 21(1):289. doi: $10.3390 /$ ijms 21010289

8. Pollheimer J, Vondra S, Baltayeva J, Beristain AG, Knofler M. Regulation of Placental Extravillous Trophoblasts by the Maternal Uterine Environment. Front Immunol (2018) 9:2597. doi: 10.3389/fimmu.2018.02597

9. Mor G, Aldo P, Alvero AB. The Unique Immunological and Microbial Aspects of Pregnancy. Nat Rev Immunol (2017) 17(8):469-82. doi: 10.1038/nri.2017.64

10. Colucci F, Moffett A, Trowsdale J. Medawar and the Immunological Paradox of Pregnancy: 60 Years on. Eur J Immunol (2014) 44(7):1883-5. doi: 10.1002/ eji.201470065

11. Williams PJ, Searle RF, Robson SC, Innes BA, Bulmer JN. Decidual Leucocyte Populations in Early to Late Gestation Normal Human Pregnancy. J Reprod Immunol (2009) 82(1):24-31. doi: 10.1016/j.jri.2009.08.001

12. Erlebacher A. Immunology of the Maternal-Fetal Interface. Annu Rev Immunol (2013) 31:387-411. doi: 10.1146/annurev-immunol-032712-100003

13. Faas MM, de Vos P. Uterine NK Cells and Macrophages in Pregnancy. Placenta (2017) 56:44-52. doi: 10.1016/j.placenta.2017.03.001

14. Parasar P, Guru N, Nayak NR. Contribution of Macrophages to Fetomaternal Immunological Tolerance. Hum Immunol (2021) 82(5):325-31. doi: 10.1016/ j.humimm.2021.02.013

15. Liu S, Diao L, Huang C, Li Y, Zeng Y, Kwak-Kim JYH. The Role of Decidual Immune Cells on Human Pregnancy. J Reprod Immunol (2017) 124:44-53. doi: 10.1016/j.jri.2017.10.045

16. Ding J, Yang C, Cheng Y, Wang J, Zhang S, Yan S, et al. Trophoblast-Derived IL-6 Serves as an Important Factor for Normal Pregnancy by Activating Stat3Mediated M2 Macrophages Polarization. Int Immunopharmacol (2021) 90:106788. doi: 10.1016/j.intimp.2020.106788

17. Swatek KN, Komander D. Ubiquitin Modifications. Cell Res (2016) 26(4):399422. doi: $10.1038 / \mathrm{cr} .2016 .39$

18. Komander D, Clague MJ, Urbe S. Breaking the Chains: Structure and Function of the Deubiquitinases. Nat Rev Mol Cell Biol (2009) 10(8):55063. doi: $10.1038 / \mathrm{nrm} 2731$

19. Kim J, Kim WJ, Liu Z, Loda M, Freeman MR. The Ubiquitin-Specific Protease USP2a Enhances Tumor Progression by Targeting Cyclin A1 in Bladder Cancer. Cell Cycle (2012) 11(6):1123-30. doi: 10.4161/cc.11.6.19550

20. Benassi B, Flavin R, Marchionni L, Zanata S, Pan Y, Chowdhury D, et al. MYC Is Activated by USP2a-Mediated Modulation of microRNAs in Prostate Cancer. Cancer Discov (2012) 2(3):236-47. doi: 10.1158/2159-8290.Cd-11-0219

21. Stevenson LF, Sparks A, Allende-Vega N, Xirodimas DP, Lane DP, Saville MK. The Deubiquitinating Enzyme USP2a Regulates the P53 Pathway by Targeting Mdm2. EMBO J (2007) 26(4):976-86. doi: 10.1038/sj.emboj.7601567

22. Shi Y, Solomon LR, Pereda-Lopez A, Giranda VL, Luo Y, Johnson EF, et al. Ubiquitin-Specific Cysteine Protease 2a (USP2a) Regulates the Stability of Aurora-A. J Biol Chem (2011) 286(45):38960-8. doi: 10.1074/jbc.M111.231498

23. Zhao Y, Wang X, Wang Q, Deng Y, Li K, Zhang M, et al. USP2a Supports Metastasis by Tuning TGF-Beta Signaling. Cell Rep (2018) 22(9):2442-54. doi: 10.1016/j.celrep.2018.02.007
24. Kim J, Alavi Naini F, Sun Y, Ma L. Ubiquitin-Specific Peptidase 2a (USP2a) Deubiquitinates and Stabilizes Beta-Catenin. Am J Cancer Res (2018) 8 (9):1823-36.

25. Livak KJ, Schmittgen TD. Analysis of Relative Gene Expression Data Using Real-Time Quantitative PCR and the $2-\Delta \Delta$ ct Method. Methods (2001) 25 (4):402-8. doi: 10.1006/meth.2001.1262

26. Scarpa E, Szabó A, Bibonne A, Theveneau E, Parsons M, Mayor R. Cadherin Switch During EMT in Neural Crest Cells Leads to Contact Inhibition of Locomotion via Repolarization of Forces. Dev Cell (2015) 34(4):421-34. doi: 10.1016/j.devcel.2015.06.012

27. Tian X, Liu Z, Niu B, Zhang J, Tan TK, Lee SR, et al. E-Cadherin/ $\beta$-Catenin Complex and the Epithelial Barrier. J BioMed Biotechnol (2011) 2011:567305. doi: $10.1155 / 2011 / 567305$

28. Ilyas M, Tomlinson IP, Rowan A, Pignatelli M, Bodmer WF. Beta-Catenin Mutations in Cell Lines Established From Human Colorectal Cancers. Proc Natl Acad Sci USA (1997) 94(19):10330-4. doi: 10.1073/pnas.94.19.10330

29. Wu K, Fan J, Zhang L, Ning Z, Zeng J, Zhou J, et al. PI3K/Akt to GSK3 $\beta / \beta$ Catenin Signaling Cascade Coordinates Cell Colonization for Bladder Cancer Bone Metastasis Through Regulating ZEB1 Transcription. Cell Signal (2012) 24(12):2273-82. doi: 10.1016/j.cellsig.2012.08.004

30. Sharma S, Godbole G, Modi D. Decidual Control of Trophoblast Invasion. Am J Reprod Immunol (2016) 75(3):341-50. doi: 10.1111/aji.12466

31. Zambuto SG, Clancy KBH, Harley BAC. A Gelatin Hydrogel to Study Endometrial Angiogenesis and Trophoblast Invasion. Interface Focus (2019) 9(5):20190016. doi: 10.1098/rsfs.2019.0016

32. Knöfler M, Haider S, Saleh L, Pollheimer J, Gamage TKJB, James J. Human Placenta and Trophoblast Development: Key Molecular Mechanisms and Model Systems. Cell Mol Life Sci (2019) 76(18):3479-96. doi: 10.1007/s00018019-03104-6

33. Graner E, Tang D, Rossi S, Baron A, Migita T, Weinstein LJ, et al. The Isopeptidase USP2a Regulates the Stability of Fatty Acid Synthase in Prostate Cancer. Cancer Cell (2004) 5(3):253-61. doi: 10.1016/s1535-6108(04)00055-8

34. Priolo C, Tang D, Brahamandan M, Benassi B, Sicinska E, Ogino S, et al. The Isopeptidase USP2a Protects Human Prostate Cancer From Apoptosis. Cancer Res (2006) 66(17):8625-32. doi: 10.1158/0008-5472.Can-06-1374

35. Allende-Vega N, Sparks A, Lane DP, Saville MK. MdmX Is a Substrate for the Deubiquitinating Enzyme USP2a. Oncogene (2010) 29(3):432-41. doi: 10.1038/onc.2009.330

36. Xiong B, Huang J, Liu Y, Zou M, Zhao Z, Gong J, et al. Ubiquitin-Specific Protease 2a Promotes Hepatocellular Carcinoma Progression via Deubiquitination and Stabilization of RAB1A. Cell Oncol (Dordr) (2021) 44 (2):329-43. doi: 10.1007/s13402-020-00568-8

37. da Silva SD, Cunha IW, Nishimoto IN, Soares FA, Carraro DM, Kowalski LP, et al. Clinicopathological Significance of Ubiquitin-Specific Protease 2a (USP2a), Fatty Acid Synthase (FASN), and ErbB2 Expression in Oral Squamous Cell Carcinomas. Oral Oncol (2009) 45(10):e134-9. doi: 10.1016/ j.oraloncology.2009.02.004

38. Qu Q, Mao Y, Xiao G, Fei X, Wang J, Zhang Y, et al. USP2 Promotes Cell Migration and Invasion in Triple Negative Breast Cancer Cell Lines. Tumour Biol (2015) 36(7):5415-23. doi: 10.1007/s13277-015-3207-7

39. Kreis N-N, Friemel A, Ritter A, Roth S, Rolle U, Louwen F, et al. Function of P21 (Cip1/Waf1/CDKN1A) in Migration and Invasion of Cancer and Trophoblastic Cells. Cancers (2019) 11(7):989. doi: 10.3390/cancers11070989

40. Luo M, Wu C, Guo E, Peng S, Zhang L, Sun W, et al. FOXO3a Knockdown Promotes Radioresistance in Nasopharyngeal Carcinoma by Inducing Epithelial-Mesenchymal Transition and the Wnt/B-Catenin Signaling Pathway. Cancer Lett (2019) 455:26-35. doi: 10.1016/j.canlet.2019.04.019

41. Kim H, Lee JH, Lee SK, Song NY, Son SH, Kim KR, et al. Chemerin Treatment Inhibits the Growth and Bone Invasion of Breast Cancer Cells. Int J Mol Sci (2020) 21(8):2871. doi: 10.3390/ijms21082871

42. Heuberger J, Birchmeier W. Interplay of Cadherin-Mediated Cell Adhesion and Canonical Wnt Signaling. Cold Spring Harb Perspect Biol (2010) 2(2): a002915. doi: 10.1101/cshperspect.a002915

43. Xie H, Tranguch S, Jia X, Zhang H, Das SK, Dey SK, et al. Inactivation of Nuclear Wnt-Beta-Catenin Signaling Limits Blastocyst Competency for Implantation. Development (2008) 135(4):717-27. doi: 10.1242/dev.015339

44. Medawar PB. Some Immunological and Endocrinological Problems Raised by the Evolution of Viviparity in Vertebrates. Symp Soc Exp Biol (1953) 7:320-37. 
45. Ning F, Liu H, Lash GE. The Role of Decidual Macrophages During Normal and Pathological Pregnancy. Am J Reprod Immunol (2016) 75(3):298-309. doi: 10.1111/aji.12477

46. Ding J, Yang C, Zhang Y, Wang J, Zhang S, Guo D, et al. M2 MacrophageDerived G-CSF Promotes Trophoblasts EMT, Invasion and Migration via Activating PI3K/Akt/Erk1/2 Pathway to Mediate Normal Pregnancy. J Cell Mol Med (2021) 25(4):2136-47. doi: 10.1111/jcmm.16191

47. Chambers M, Rees A, Cronin JG, Nair M, Jones N, Thornton CA. Macrophage Plasticity in Reproduction and Environmental Influences on Their Function. Front Immunol (2020) 11:607328. doi: 10.3389/fimmu.2020.607328

48. Lafontaine L, Chaudhry P, Lafleur M-J, Van Themsche C, Soares MJ, Asselin E. Transforming Growth Factor Beta Regulates Proliferation and Invasion of Rat Placental Cell Lines1. Biol Reprod (2011) 84(3):553-9. doi: 10.1095/ biolreprod.110.086348

49. Shooner C, Caron PL, Fréchette-Frigon G, Leblanc V, Déry MC, Asselin E. TGF-Beta Expression During Rat Pregnancy and Activity on Decidual Cell Survival. Reprod Biol Endocrinol (2005) 3:20. doi: 10.1186/1477-7827-3-20

50. Brooks SA, Martin E, Smeester L, Grace MR, Boggess K, Fry RC. miRNAs as Common Regulators of the Transforming Growth Factor (TGF)- $\beta$ Pathway in the Preeclamptic Placenta and Cadmium-Treated Trophoblasts: Links Between the Environment, the Epigenome and Preeclampsia. Food Chem Toxicol (2016) 98:50-7. doi: 10.1016/j.fct.2016.06.023

51. Chaiworapongsa T, Romero R, Korzeniewski SJ, Chaemsaithong P, Hernandez-Andrade E, Segars JH, et al. Pravastatin to Prevent Recurrent Fetal Death in Massive Perivillous Fibrin Deposition of the Placenta (MPFD). J Maternal Fetal Neonatal Med Off J Eur Assoc Perinatal Med Fed Asia
Oceania Perinatal Soc Int Soc Perinatal Obstet (2016) 29(6):855-62. doi: 10.3109/14767058.2015.1022864

52. Ouahoud S, Jacobs RJ, Peppelenbosch MP, Fühler GM, Heijmans J, Diks S, et al. Kinome-Wide Analysis of the Effect of Statins in Colorectal Cancer. Br J Cancer (2021) 124(12):1978-87. doi: 10.1038/s41416-021-01318-9

53. Ipsa E, Cruzat VF, Kagize JN, Yovich JL, Keane KN. Growth Hormone and Insulin-Like Growth Factor Action in Reproductive Tissues. Front Endocrinol (Lausanne) (2019) 10:777. doi: 10.3389/fendo.2019.00777

Conflict of Interest: The authors declare that the research was conducted in the absence of any commercial or financial relationships that could be construed as a potential conflict of interest.

Publisher's Note: All claims expressed in this article are solely those of the authors and do not necessarily represent those of their affiliated organizations, or those of the publisher, the editors and the reviewers. Any product that may be evaluated in this article, or claim that may be made by its manufacturer, is not guaranteed or endorsed by the publisher.

Copyright (C) 2021 Wang, Ding, Zhang, Chen, Yan, Zhang and Yin. This is an openaccess article distributed under the terms of the Creative Commons Attribution License (CC BY). The use, distribution or reproduction in other forums is permitted, provided the original author(s) and the copyright owner(s) are credited and that the original publication in this journal is cited, in accordance with accepted academic practice. No use, distribution or reproduction is permitted which does not comply with these terms. 